\title{
Voriconazole Induced Severe Liver Toxicity Post Keratitis
}

Dpen Access

\author{
Fahad I. Al-Saikhan* \\ College of Pharmacy, Salman Bin Abdulaziz University, Al-Kharj, KSA
}

\begin{abstract}
The case involved a 72-year old Saudi female patient who was treated for fungal keratitis. Aspergillus species was confirmed. The patient showed mild liver impairment upon admission. Antimicrobial eye drop solutions which comprise the medicines for the treatment regimen included antibiotics (gentamicin, cefazoline, ceftazidine, and moxifloxacin) and antifungal (amphotericin B and, voriconazole) that were started on day one, as well as, voriconazole systemic. On day 9 of the hospitalization, the liver function tests (LFTs) revealed significantly higher values compared to day zero. All medicines were continued at their usual doses and no adverse drug reaction was reported. On Day 13, the patient experienced abdominal pain, hallucination, nausea, tachycardia, and vomiting, in addition to elevated LFTs. At this time, oral voriconazole was discontinued. Patient was discharged with no further reports. An analysis of adverse drug event (ADE) report was performed and results showed that systemic voriconazole is the possible cause of the significant increase in LFTs and the manifested symptoms of liver toxicity.
\end{abstract}

Keywords: Voriconazole, keratitis, Aspergillus, liver toxicity.

\section{INTRODUCTION}

72-year old Saudi female in this case was under treatment for microbial keratitis. Initial tests upon admission showed mild impairment of the liver. LFTs evaluation revealed mild increase as follow: Gamma-glutamyltransferase (GGT) 147 Unit/Liter (U/L), Alanine transaminase (ALT) $69 \mathrm{U} / \mathrm{L}$, and Alkaline phosphatase (ALP) 147 U/L. Aspergillus species was confirmed. However, no susceptibility testing was conducted for voriconazole (an antifungal) before it was administered in the hospital.

The treatment regimen included: antimicrobial eye drops using the antibiotics gentamicin, cefazoline, ceftazidine, and moxifloxacin which all initiated on day zero then discontinued on day two except for the later. Antifungals amphotericin B eye drops, voriconazole eye drops, as well as oral voriconazole 200 milligram (mg) were commenced on day one. However, no sensitivity testing was conducted. On day 9 , the patient's laboratory results showed a significant increase in the LFTs as follows: GGT $253 \mathrm{U} / \mathrm{L}$, ALT $139 \mathrm{U} / \mathrm{L}$, and ALP 197 U/L. No ADE report was submitted and none of the medicines included in the treatment regimen (voriconazole topical and system, amphotericin B topical, and moxifloxacin topical) were discontinued until Day 13. On this date, the patient experienced abdominal pain, hallucination, nausea, tachycardia, and vomiting, in addition to substantial elevation in LFTs (GGT $534 \mathrm{U} / \mathrm{L}$, ALT $148 \mathrm{U} / \mathrm{L}$, and ALP $278 \mathrm{U} / \mathrm{L}$ ). This paper discusses the possible causes of the considerable increase in specific liver enzymes and other symptoms experienced by the patient. It is also of noteworthy to trace any lapses which may have transpired, and their implications in our patient.

*Address correspondence to this author at the College of Pharmacy, Salman Bin Abdulaziz University, Al-Kharj, KSA; Tel: +966 115886074; Fax:+966 115886001; Email: fsaikhan@hotmail.com

\section{DISCUSSION}

Confirmation of the initial diagnosis for the presenting symptoms of the case is an important process in the treatment to ensure that the appropriate medicines are prescribed.

There are two primary causes of raised plasma GGT and ALP: either liver disease or induction of microsomal enzymes by alcohol or most likely by drugs [1]. A review of drug adverse reactions may provide a more meaningful analysis of the case grounded on research evidence that:

This is a Saudi female that is not consuming alcohol therefore less likely to be the cause of raised LFTs. In addition, women typically consume less alcohol than men when they drink, drink alcohol less frequently, and are less likely to develop alcohol related problems than men [2].

It is reported that systemic administration of amphotericin B and gentamicin could be a factor in elevation of LFTs [3]. However, in this case both were administered topically. And gentamicin was administered only from day zero to day two.

There were concerns pertaining to liver toxicity related to the administration of systemic voriconazole. However, a series of tests has already been performed in response to concerns articulated by some sectors of the medical community. Results of these tests, particularly, three randomized trials of voriconazole revealed that the frequency of abnormalities from LFTs after administration of systemic voriconazole was not significantly different from those of systemic amphotericin [4].

Additionally, a review of unpublished studies which employed regression analysis on a total of 3000 samples from 1000 patients in 10 trials accounted for a weak and inconsistent association of abnormal results of liver function tests with plasma concentrations of voriconazole after systemic administration. A number of studies provide evidence of 
major adverse effects from systemic voriconazole including elevated hepatic enzymes among $\geq 20 \%$ of patients $[5,6]$. It was, however, recommended that individual dosage among patients be modified based on voriconazole plasma concentration levels [4].

In our case, voriconazole $200 \mathrm{mg}$ orally every 12 hours and voriconazole $1 \%$ drops on the left eye every hour were prescribed. With reference to the standard guidelines on administering voriconazole, [7] it was observed that the loading dose $200 \mathrm{mg}$ orally for two doses was given to the patient, which presumably weighs less than 40 kilogram $(\mathrm{kg})$. But continued on maintenance dose of $200 \mathrm{mg}$ every twelve hours for 13 days. The dosage administered to our patient who showed mild impairment of the liver function was not modified or customized based on the patient's condition upon admission. Moreover, based on the aforementioned guidelines prepared from the Infectious Diseases Society of America, in patients with mild to moderate hepatic impairment (Child-Pugh Class A and B), $100 \mathrm{mg}$ of voriconazole may be administered every 12 hours for patients who weigh $>40 \mathrm{~kg}$ and $50 \mathrm{mg}$ every 12 hours for patients who weigh $<40 \mathrm{~kg}$. [7].

There is, therefore, reason to believe that the dosage given to the patient did not conform with the guidelines of the healthcare facility where the patient is being treated. This non-conformance to clinical protocol could most probably be the reason for the spike in patient's LFTs (GGT and ALP) above normal levels. In spite of the high GGT and ALP reported from the patient's laboratory result, the patient continued receiving the same dose of systemic voriconazole until Day 13 of confinement. Besides the internal clinical guidelines from the healthcare facility on the administration of systemic voriconazole, there is research evidence that adjustments are necessary for systemic voriconazole dosage among patients known to have mild or moderate hepatic impairment $[8,9]$.

As indicated in our case, patient suffered from the following symptoms on Day 13: abdominal pain, hallucination, nausea, tachycardia, and vomiting, in addition to elevated LFTs. Nausea and vomiting were reported among the adverse effects of voriconazole [4]. Abdominal pain, hallucination, and tachycardia were also known adverse effects of voriconazole [10]. Thus, patient symptoms were manifested in the central nervous system, together with cardiovascular, gastro-intestinal problems, and significantly increased GGT and ALP.

There was apparent lapse in the management of patient safety when the bedside nurse did not call the attention of concerned authorities regarding the adverse effect of the treatment regimen on the patient. The ophthalmologist should have also seen the patient chart with the result of the LFT and the elevated GGT and ALP. Generally accepted protocol in this regard is for the attending nurse or the bedside nurse to fill in an ADE report. Which was a routine procedure at this facility to report any ADE due to use of any medication? The reported side effect for our case was only submitted on day 13 .

\section{CONCLUSION}

In retrospect, health care practitioners should be aware about the seriousness of liver injury from systemic voriconazole administration, which may include mild or transient elevation of LFTs or hepatic impairment at the worst. There have also been reports of voriconazole-induced acute liver failure [11]. Such complications might require liver transplant and may even cause death. Caution should always be taken when voriconazole is administered to patients. Dose adjustment is generally recommended for patients with hepatic impairment. The health care team should always be on the lookout for manifestations of liver toxicity.

\section{CONFLICT OF INTEREST}

The author(s) confirm that this article content has no conflicts of interest.

\section{ACKNOWLEDGEMENT}

Declared none.

\section{REFERENCES}

[1] Koay E, Noah W. A Primer of Chemical Pathology. London: World Scientific, 1996.

[2] Green C. Gender and use of substance abuse treatment services. National institute on alcohol abuse and alcoholism (no date). Available from: http://pubs.niaaa.nih.gov/publications/ arh291/5562.htm [cited: $2^{\text {nd }}$ Apr 2013].

[3] Kevin Sullivan, Ed. Nurse's Drug Handbook. $12^{\text {th }}$ ed. Burlington: Jones \& Bartlett's Learning, 2013.

[4] Groll A, Hedwig K, Gary W. Antifungal drugs. Side Effects of Drugs. Annual $28^{\text {th }}$ ed. San Diego: Elsevier 2005.

[5] Jeu LA, Piacenti FJ, Lyakhovetskiy AG, Fung HB. Voriconazole. Clin Ther 2003; 25(5): 1321.

[6] Hariprasad S, William M, Eric H, et al. Determination of vitreous, aqueous, and plasma concentration of orally administered voriconazole in humans. Arch Ophthalmol Chic 2004; 122(1): 41-7.

[7] Walsh T, Elias A, David D, et al. Treatment of Aspergillosis: clinical practice guidelines of the infectious diseases society of America. Clin Infect Dis 2008; 46(3): 327-60.

[8] Weiler S, Zoller H, Graziadei I, et al. Altered pharmacokinetics of voriconazole in a patient with liver cirrhosis. Antimicrob Agents Chemother 2007; 51: 3459.

[9] Lat A, Thompson G. Update on the optimal use of voriconazole for invasive fungal infections. Infect Drug Resist 2011; 4: 45.

[10] Karch A. Lippincott's Nursing Drug Guide. PA, USA: Lippincott Williams \& Wilkins 2013.

[11] National Library of Medicine. Drug record: Voriconazole. Liver tox database. Available from: http://ivertox.nlm.nih.gov/ Voriconazole.htm [cited: $2^{\text {nd }}$ Apr 2013].

This is an open access article licensed under the terms of the Creative Commons Attribution Non-Commercial License (http://creativecommons.org/licenses/by-nc/3.0/) which permits unrestricted, non-commercial use, distribution and reproduction in any medium, provided the work is properly cited. 